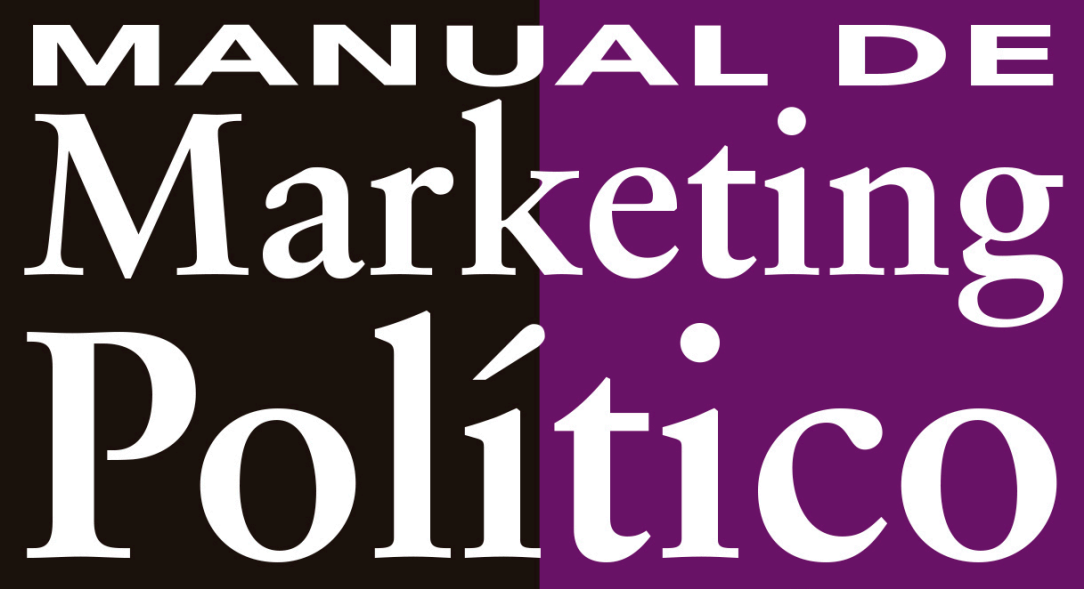

ESTRATEGIAS PARA UNA CAMPAÑA EXITOSA

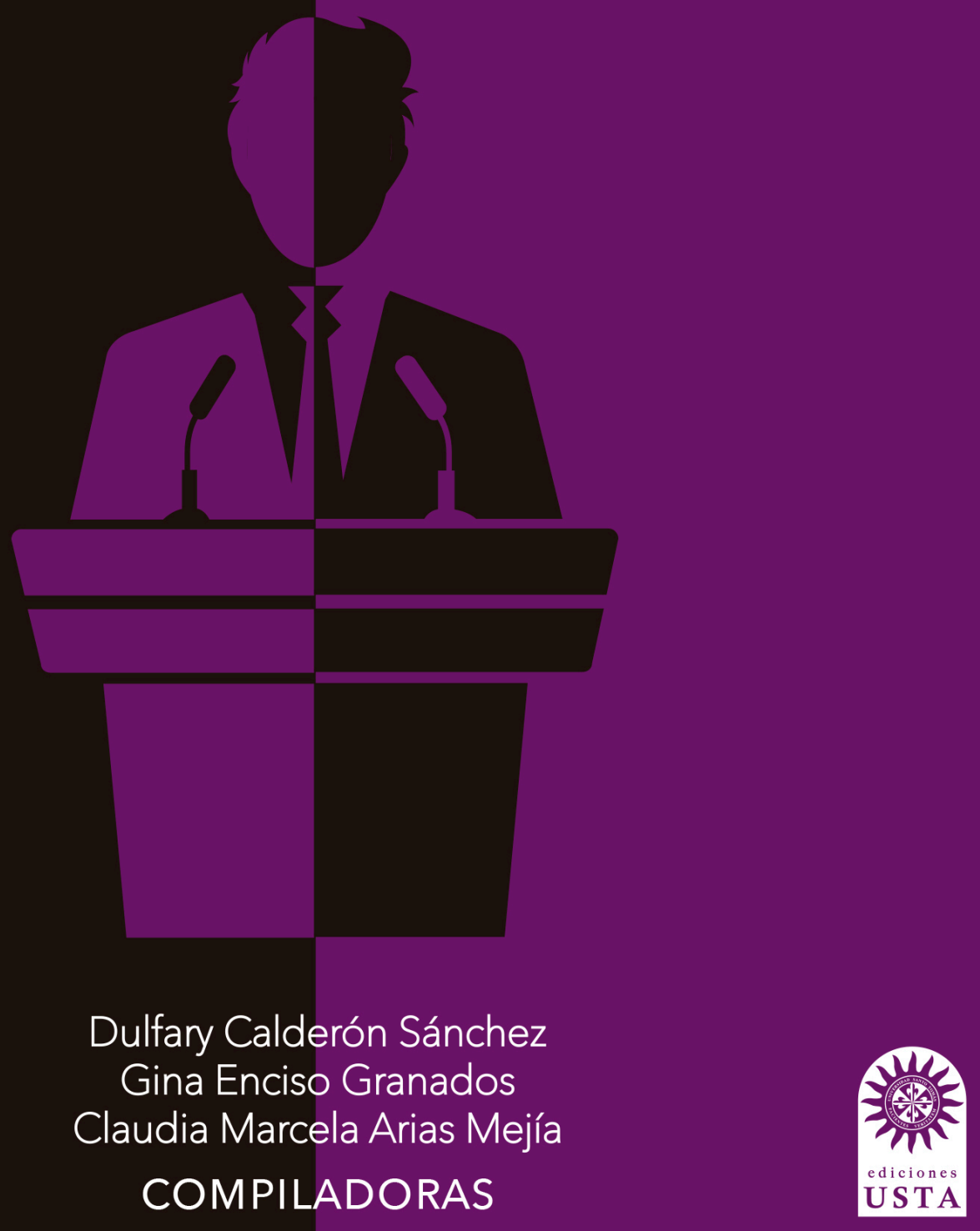




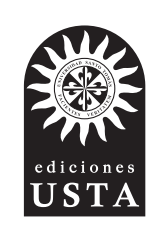

\section{Manual de \\ Marketing Político}

ESTRATEGIAS PARA UNA CAMPAÑA EXITOSA

Dulfary Calderón Sánchez

Gina Enciso Granados

Claudia Marcela Arias Mejía

COMPILADORAS 
Manual de Marketing Político /Gina Enciso Granados [y otros cuatro], compiladoras Claudia Marcela Arias, Dulfary Calderón Sánchez, Gina Enciso Granados, Bogotá: Universidad Santo Tomás, 2017.

117 páginas, ilustraciones, graficas.

Incluye referencias bibliográficas

ISBN 978-958-782-011-9

E- ISBN 978-958-782-012-6

1. Ciencia política 2. Comunicación en política 3. Comunicación visual 4. Análisis del discurso I. Universidad Santo Tomás (Colombia).

CDD 324.73

CO-BoUST

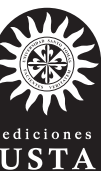

(C) Dulfary Calderón Sánchez, Gina Enciso Granados, Daniel Arturo Palma Álvarez, Paula Camila Amador Cardona, Claudia Marcela Arias Mejía

\section{Compiladoras}

Claudia Marcela Arias Mejía, Dulfary Calderón Sánchez, Gina Enciso Granados

(C) Universidad Santo Tomás, 2017

Ediciones USTA Carrera 9 n. ${ }^{\circ}$ 51-11

Bogotá, D. C., Colombia

Teléfonos: (+571) 5878797 ext. 2991

editorial@usantotomas.edu.co

http://www.ediciones.usta.edu.co

Coordinación de libros: Karen Grisales Velosa

Asistente editorial: Andrés Felipe Andrade

Corrección de estilo: Pamela Montealegre

Diseño y diagramación: Alexandra Romero Cortina

Impresión: Image Printing Ltda.

Hecho el depósito que establece la ley

ISBN 978-958-782-011-9

E-ISBN 978-958-782-012-6

Se prohíbe la reproducción total o parcial de esta obra, por cualquier medio, sin la autorización expresa de Ediciones USTA.

Impreso en Colombia • Printed in Colombia

Primera edición, 2017

Primera reimpresión, 2018 


\section{Tabla de contenido}

Presentación IX

Alberto Castillo Castañeda

Ricardo Hoyos Ballesteros

Piedad Rocío Gómez Castillo

Primera Parte. Mercadeo i I

Introducción al marketing político $\quad$ I3

Gina Enciso Granados

SEgunda PARTE. LA IMPORTANCIA DEL Discurso

Y DEL MENSAJE POLÍTICO

5 I

Definición del concepto de discurso político:

algunas consideraciones teórico-prácticas

53

Daniel Arturo Palma Álvarez

La propuesta política como difusión y mensaje

73

Dulfary Calderón SÁNCheZ

TERCERA PARTE. LOS ELEMENTOS DE LA COMUNICACIÓN VISUAL Y LA IDENTIDAD GRÁFICA

¿Todo entra por los ojos? Comunicación visual para la política $\quad 85$

Paula Amador Cardona

La identidad de marca en campañas políticas 99

Claudia Marcela Arias Mejía

\section{Anexo}

Guía para la planificación de la campaña 



\section{Presentación}

T a Universidad Santo Tomás, en cabeza del Rector General, Fr. Juan UUbaldo López Salamanca, O. P., el Vicerrector Académico General, Fr. Mauricio Antonio Cortés Gallego, O. P., los Decanos de División, Fr. Carlos Arturo Díaz, O. P., Fr. Alberto Rene Rodríguez, O. P. y Fr. Esneider Claros Castro, O. P. a través de su conocimiento y experiencia en la academia, han propiciado escenarios de comunicación e integración entre las Facultades gestoras de este proyecto para responder de manera crítica, creativa y propositiva a la generación de un Marketing Político con un enfoque humanista.

Dado el contexto social y político tan complejo en que se desarrollan las contiendas electorales en nuestro país y por tanto, los desafíos que implican las nuevas herramientas de campaña, desde la Universidad Santo Tomás se propició un escenario interdisciplinario con el objetivo de aportar, desde la academia a la formación de nuevos líderes con sentido humano capaces de solventar la aplicabilidad del marketing político como herramienta para ser competitivos en el entorno, sin dejar a un lado la perspectiva del bien común.

En este orden de ideas, nos complace entregar a la comunidad académica, social y política el presente documento como una herramienta académica que condensa algunos elementos aportados desde las facultades de Diseño Gráfico, Mercadeo y Gobierno y Relaciones 
Internacionales a partir de la experiencia obtenida con un grupo de estudiante en coordinación con docentes investigadores que desarrollaron asesoría, acompañamiento y consultoría de manera gratuita en el tema de marketing político y de gobierno a candidatos para edil en el Distrito Capital durante el periodo 2016-2019.

La línea argumentativa del texto en mención permite identificar los elementos estructurales que deben hacer parte de una campaña electoral, así de manera sencilla y práctica, se le permite al lector o posible candidato, conocer los alcances, límites y directrices necesarios en el desempeño de un proceso electoral desde el ámbito local hasta el nacional.

El documento se encuentra distribuido en tres partes; en primer lugar, se encuentra el área de Marketing, donde se desarrollan los temas de investigación de mercados, E-Marketing, comunicación, publicidad \& relaciones públicas y Branding. En segundo lugar, se aborda el área de Gobierno, en donde se profundizan los temas de oralidad y análisis del discurso, lenguaje y construcción política y análisis de campañas políticas. Finalmente, en tercer lugar, se profundiza en el diseño gráfico, la comunicación visual, la identidad gráfica, la fotografía y las campañas promocionales en el ámbito político.

Así, estimado lector, ponemos a su disposición este primer aporte científico-académico que se genera a partir de la conjugación de las tres disciplinas académicas que fundamentan y dotan de un marco referencial para permitir de manera sencilla acceder a las herramientas, elementos y factores prácticos en la construcción de su propia campaña.

Alberto Castillo Castañeda Decano Facultad Gobierno y Relaciones Internacionales

Ricardo Hoyos Ballesteros

Decano Facultad de Mercadeo

Piedad Rocío Gómez Castillo Decana Facultad de Diseño Gráfico 